\title{
Urethral Melanoma
}

National Cancer Institute

\section{Source}

National Cancer Institute. Urethral Melanoma. NCI Thesaurus. Code C159665.

A rare melanoma that arises from the urethral mucosa. 